\title{
Application of a virtual inerter in active vibration control using inertial actuators
}

\author{
N. Debattisti ${ }^{a}$, M. L. Bacci ${ }^{a}$, S. Cinquemani ${ }^{a}$ \\ ${ }^{a}$ Politecnico di Milano, Dipartimento di Meccanica, Via La Masa 1, 20156 Milano, Italy.
}

\begin{abstract}
In this paper the application of an inerter to a control system based on inertial actuators is investigated. Since the dynamics of this kind of actuators affects the stability of the controlled system, the application of the inerter aims to limit instability problems by shifting down the resonance frequency of the actuator. The interaction of this passive element with the other vibration modes of the structure under control is not negligible. The inerter should be physically placed in parallel with the elastic suspension of the actuator and the transducer; in this paper, the inerter behavior is simulated by the inertial actuator through an acceleration feedback. First, the study is carried out on a two degrees-of-freedom model and stability considerations are made; then, the approach is implemented on the Finite Element model of a clamped-clamped beam which is also used as experimental test rig. Finally, the proposed solution is validated with experimental results.
\end{abstract}

Keywords: Vibration control, Inertial actuators, Control algorithms, Automation

\section{INTRODUCTION}

Among the different solutions adopted in structural vibrations control, active control systems can be a valid alternative to passive dampers, as they provide higher performance, especially for low-frequency applications. One of the most effective strategies which is adopted in vibration control is to increase the equivalent damping of the system through the so called Skyhook damping technique. ${ }^{1}$

This kind of control system is only conditionally stable due to the particular actuator dynamics. The stability of the controller is then related to the natural frequency of the actuator, which must be as low as possible compared to the frequency of the first vibration mode of the host structure. ${ }^{2}$ However, a low resonance is difficult to achieve in practice, because a soft suspension will cause large static deflection due to gravity and will increase the risk that the inertial mass could hit the ends-stop, potentially leading to instability phenomena. ${ }^{3}$ Usually, this kind of actuators are used for application in which the previous condition is fulfilled.

In this paper, the influence of an inerter ${ }^{4,5}$ on the fundamental resonance frequency of the actuator is investigated. This study has been carried out by taking a cue from the approach developed in a previous work ${ }^{6}$ and by replacing the physical inerter with a virtual one, whose behaviour has been simulated by the control force generated by the actuator. The aim is to lower the resonance frequency of the inertial actuator in order to limit instability problems mentioned before. The solution has been validated considering 1) a two degrees of freedom lumped parameters system which simulates the actuator dynamic and the behaviour of a single vibration mode of the structure and 2) the FEM model of a clamped-clamped beam, which describes the behaviour of the experimental test rig.

The most used control architectures require a complex setup of all the components involved in the control task: many sensors and actuators have to be installed and wired to a real-time board, which manages the input signals coming from sensors and, through the implementation of the control logic, the output signals to provide to the actuators. To overcome this problem, a stand-alone device has been developed, which is fitted with all the necessary elements to work in an autonomous way: there are sensors for the data acquisition, an microcontroller in which the control algorithm is implemented and the actuator, driven by the board. This kind of device has been used to implement the control logic and then to perform the experimental tests of the proposed solution.

Further author information: (Send correspondence to Simone Cinquemani) E-mail: simone.cinquemani@polimi.it Telephone: +39 (0)2 2399.8454 


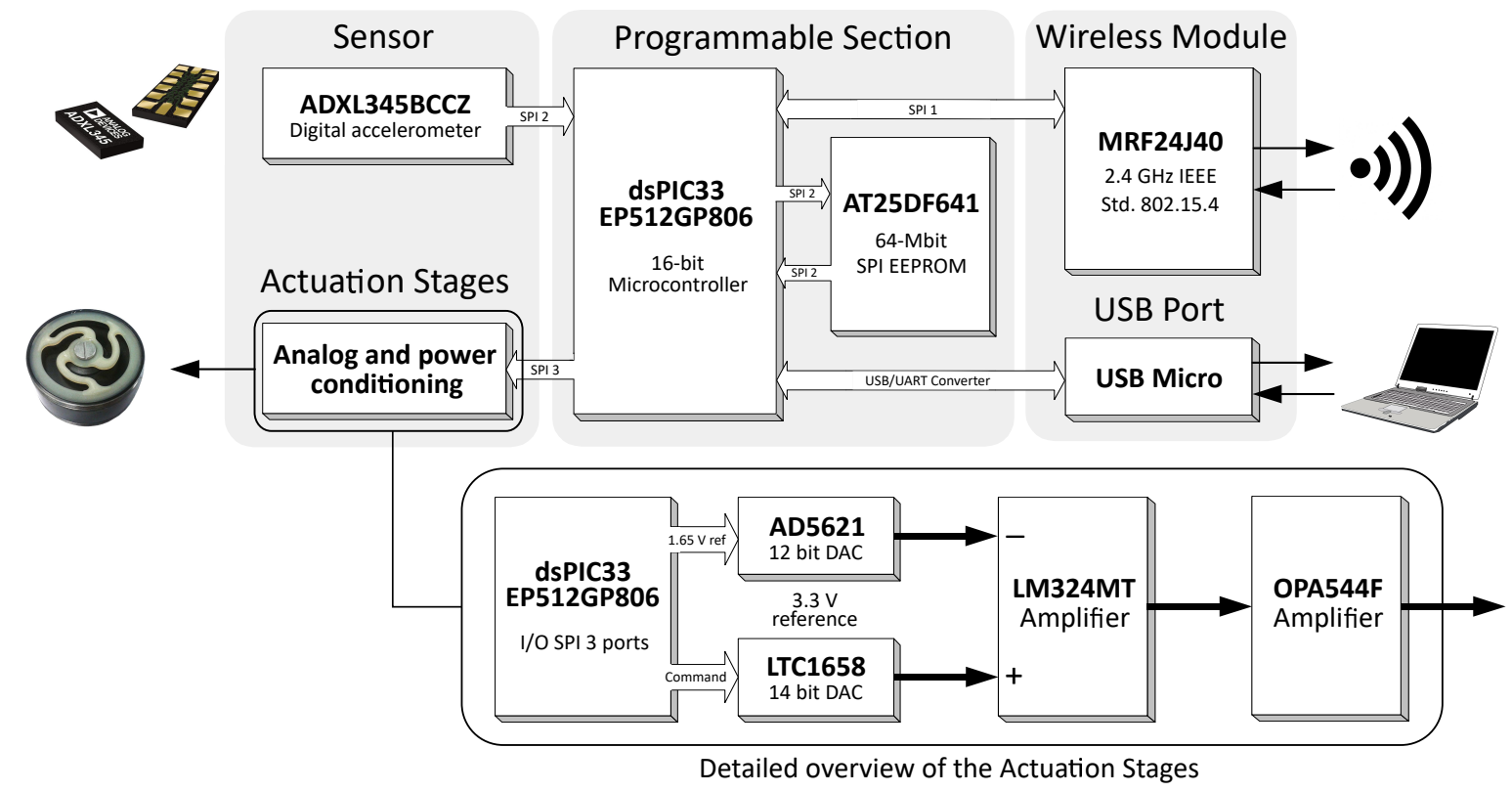

Figure 1: Block diagram which represents how the board is designed

The paper is structured as follows. In Section 2 the features of the stand-alone device are presented, while in Section 3 the mathematical formulation of the inerter behaviour embedded with the control algorithm is derived. In Section 4 the experimental results obtained on a large vibrating system are shown. Finally, conclusions are drawn in Section 5.

\section{THE STAND-ALONE DEVICE}

The developed device ${ }^{7,8}$ is able to carry out operations of vibration control in an autonomous way. Two MEMS digital accelerometer are available: one is placed on the fixed frame of the device and the other on the suspended mass of the actuator. The first sensor measures the acceleration of the structure, which will be used to compute the control signal, while the second one measures the acceleration of the actuator, in order to get information about its dynamics during the control action.

These signals are acquired by the microprocessor, the main element of the electronic board in which different control logics can be implemented. ${ }^{9-11}$ The input signals are filtered in order to cancel out the static acceleration and other contributions which can lead to instability problems; the output of the control algorithm is the command signal: in order to drive the actuator, this signal must be converted from digital to analog, conditioned and amplified by the analog and power stages (see the Actuation stages in Fig. 1). The actuation system consists in a magnetodynamics inertial actuator, which can be modeled as a mass-spring-damper 1-DOF system excited by means of an electromagnetic force. ${ }^{12-14}$ The dynamics of this kind of actuators ${ }^{15}$ must necessarily be considered during the design phase of the control logic; under their resonance frequency, inertial actuators generate a force with a small module and which is in counter-phase with respect to the vibration signal, leading to instability problems. ${ }^{16-18}$ The electronic board is also provided with a wireless module, which allows the communication with the other stand-alone devices (both transmission and reception). The wired communication with the computer is needed for the firmware loading and for the transmission of commands in order to manage the operations of the board. Moreover, it is possible to read and acquire data from sensors.

\section{NUMERICAL SIMULATIONS}

\subsection{Two degrees-of-freedom model}

The 2-dof model represents the dynamics of one vibration mode of the structure and the dynamics of the inertial actuator (Fig. 2); both of them are modeled as a mass-spring-damper system $\left(m_{1}-k_{1}-c_{1}\right.$ and $m_{a}-k_{a}-c_{a}$ 


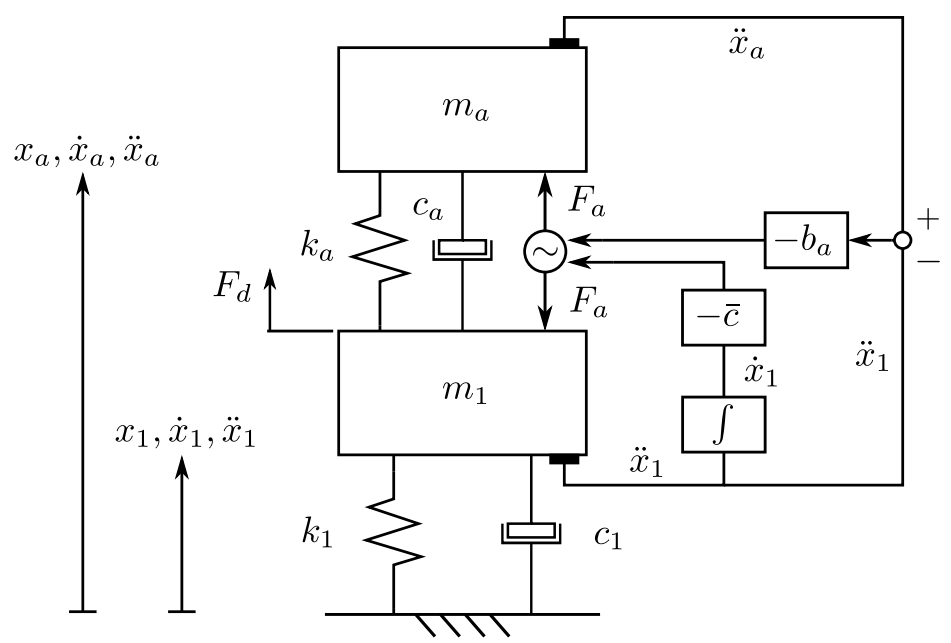

Figure 2: Schematics of the control unit model used in simulations

respectively). An external force $F_{d}$ is applied to the primary structure. The parameters of the actuator have been chosen like the real ones, while the parameters of the structure (in particular the mass $m_{1}$ ) have been chosen in order to better highlight how an inertial actuator behaves when it is driven to reduce the vibration of the primary structure. In this study the control action is designed not only to increase the damping of the system, but also to simulate the presence of a physical inerter placed in parallel with the suspension of the inertial actuator. Like a damper or a spring, which produce a force related to the relative speed or relative displacement, an inerter is a passive element which generates a force proportional to the relative acceleration between two moving masses. ${ }^{19}$ Therefore, it is possible to reproduce its behaviour by introducing a feedback loop with the acceleration of the primary structure $\ddot{x}_{1}$ and the acceleration of the suspended mass of the actuator $\ddot{x}_{a}$. The control law can be written as follows:

$$
F_{a, \text { inerter }}(t)=-b_{a}\left[\ddot{x}_{a}(t)-\ddot{x}_{1}(t)\right]
$$

in which the gain of the loop, $b_{a}$, represents the virtual inertance (measured in kilogram). In this theoretical example, stability or performance considerations related to the control part which simulates the presence of the inerter are not initially made; this passive element will be treated as if it were physically present. In the first part it is interesting only to investigate the effect of a pure inerter of the controlled system; afterwards, the whole dynamic will be taken into account.

The actuator also produces a sky-hook active force $F_{a, s k y-h o o k}$ proportional to the absolute velocity of the modal mass $m_{1}$, via the control gain $\bar{c}$. The total control force thus becomes:

$$
F_{a}(t)=F_{a, s k y-h o o k}(t)+F_{a, \text { inerter }}(t)=-\bar{c} \dot{x}_{1}(t)-b_{a}\left[\ddot{x}_{a}(t)-\ddot{x}_{1}(t)\right]
$$

The equation of motion of the system can be written in a matrix form:

$$
\begin{aligned}
{\left[\begin{array}{cc}
m_{1} & 0 \\
0 & m_{a}
\end{array}\right]\left[\begin{array}{l}
\ddot{x}_{1}(t) \\
\ddot{x}_{a}(t)
\end{array}\right]+\left[\begin{array}{cc}
c_{1}+c_{a} & -c_{a} \\
-c_{a} & c_{a}
\end{array}\right]\left[\begin{array}{c}
\dot{x}_{1}(t) \\
\dot{x}_{a}(t)
\end{array}\right]+} \\
+\left[\begin{array}{cc}
k_{1}+k_{a} & -k_{a} \\
-k_{a} & k_{a}
\end{array}\right]\left[\begin{array}{c}
x_{1}(t) \\
x_{a}(t)
\end{array}\right]=\left[\begin{array}{c}
F_{d}(t) \\
0
\end{array}\right]+\underline{F}_{a}(t)
\end{aligned}
$$

where the vector $\underline{F}_{a}(t)$ is defined as:

$$
\underline{F}_{a}(t)=\left[\begin{array}{c}
-\bar{c} \dot{x}_{1}(t)+b_{a}\left[\ddot{x}_{a}(t)-\ddot{x}_{1}(t)\right] \\
\bar{c} \dot{x}_{1}(t)-b_{a}\left[\ddot{x}_{a}(t)-\ddot{x}_{1}(t)\right]
\end{array}\right]
$$

since the force generated by the actuator is applied both on the suspended mass and the primary structure. The 
terms in Eq. 4 can be brought to the left side of the matrix equation, resulting in:

$$
\begin{array}{r}
{\left[\begin{array}{cc}
m_{1}+b_{a} & -b_{a} \\
-b_{a} & m_{a}+b_{a}
\end{array}\right]\left[\begin{array}{c}
\ddot{x}_{1}(t) \\
\ddot{x}_{a}(t)
\end{array}\right]+\left[\begin{array}{cc}
c_{1}+c_{a}+\bar{c} & -c_{a} \\
-c_{a}-\bar{c} & c_{a}
\end{array}\right]\left[\begin{array}{c}
\dot{x}_{1}(t) \\
\dot{x}_{a}(t)
\end{array}\right]+} \\
+\left[\begin{array}{cc}
k_{1}+k_{a} & -k_{a} \\
-k_{a} & k_{a}
\end{array}\right]\left[\begin{array}{c}
x_{1}(t) \\
x_{a}(t)
\end{array}\right]=\left[\begin{array}{c}
F_{d}(t) \\
0
\end{array}\right]
\end{array}
$$

Considering the column vectors:

$$
\underline{x}(t)=\left[\begin{array}{ll}
x_{1}(t) & x_{a}(t)
\end{array}\right]^{T} ; \quad \underline{F}(t)=\left[\begin{array}{cc}
F_{d}(t) & 0
\end{array}\right]^{T}
$$

Eq. 5 becomes:

$$
[M] \underline{\ddot{x}}(t)+[C] \underline{\dot{x}}(t)+[K] \underline{x}(t)=\underline{F}(t)
$$

where $M, C$ and $K$ are the mass, the damping and the stiffness matrices respectively. It is important to notice that due to the feedback loop the damping matrix $C$ is non-symmetric. This means that the control system will be only conditionally stable.

Considering an harmonic disturbance and applying the Laplace Transform, Eq. 7 becomes:

$$
\underbrace{\left([M] s^{2}+[C] s+[K]\right)}_{[A(s)]} \underline{X}(s)=\underline{F}(s)
$$

where $A(s)$ is the so called "dynamic stiffness matrix". By inverting the matrix $A$, the solution of Eq. 8 can be obtained:

$$
\underline{X}(s)=[A(s)]^{-1} \underline{F}(s)
$$

Then, by deriving in time the last equation, the solution in terms of velocities is obtained:

$$
\underline{\dot{X}}(s)=[\tilde{A}(s)] \underline{F}(s)
$$

$\tilde{A}(s)=s \cdot A^{-1}(s)$ is the so called "mobility matrix". In this case, it is defined as follows:

$$
[\tilde{A}(s)]=\frac{s}{\operatorname{det}[A(s)]} \cdot\left[\begin{array}{cc}
\left(m_{a}+b_{a}\right) s^{2}+c_{a} s+k_{a} & b_{a} s^{2}+c_{a} s+k_{a} \\
b_{a} s^{2}+\left(c_{a}+\bar{c}\right) s+k_{a} & \left(m_{1}+b_{a}\right) s^{2}+\left(c_{1}+c_{a}+\bar{c}\right) s+k_{1}+k_{a}
\end{array}\right]
$$

Then, it is possible to compute the steady-state response of the system as:

$$
\begin{aligned}
& \tilde{A}_{11}(s)=\frac{\dot{x}_{1}}{F_{d}}=\frac{\left(m_{a}+b_{a}\right) s^{3}+c_{a} s^{2}+k_{a} s}{\operatorname{det}[A(s)]} \\
& \tilde{A}_{21}(s)=\frac{\dot{x}_{a}}{F_{d}}=\frac{b_{a} s^{3}+\left(c_{a}+\bar{c}\right) s^{2}+k_{a} s}{\operatorname{det}[A(s)]}
\end{aligned}
$$

In Fig. 3 is shown the block diagram of the controlled system, in which $L(s)$ is the open-loop FRF defined as follows:

$$
L(s)=\tilde{A}_{21,0}(s)-\tilde{A}_{11,0}(s)
$$

$\tilde{A}_{11,0}(s)$ and $\tilde{A}_{21,0}(s)$ are obtained from Eqs. 12 and 13 by setting $\bar{c}=0$.

In Fig. 4 are shown the Bode and the Nyquist diagrams of the open-loop FRF $L(s)$. The dashed line represents the open-loop FRF for the system configuration without the inerter while the solid line represents the open-loop FRF considering with the virtual inerter introduced in this section. Moreover, the presence of the inerter (as it can be considered as an additional mass) affects the value of the damping ratio, which is defined as follows:

$$
\zeta_{a}=\frac{c_{a}}{2 \sqrt{k_{a}\left(m_{a}+b_{a}\right)}}
$$




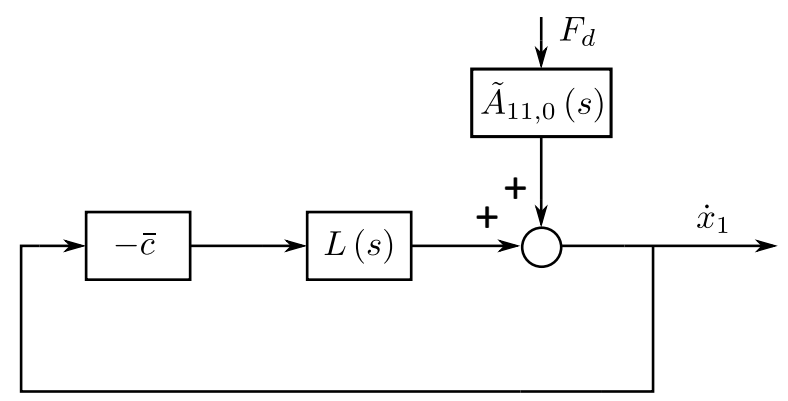

Figure 3: Block diagram of the control system

As expected, looking at the dashed line, the magnitude of the open-loop FRF shows two peaks at the resonance frequencies of both the actuator and the primary structure. The two resonant responses are out of phase, thus the low frequency portion of the open-loop FRF locus shown The Nyquist diagram in Fig. 4b shows a circle in the low frequency region, which corresponds to the first resonance; this means that the stability of the closed loop is conditioned by the value of the feedback loop gain.

In Fig. 5 are represented the FRFs $\left(\ddot{x}_{1} / F_{d}\right)$ of the system without (a) and with (b) the inerter. The dashed line curve shows that, by increasing the feedback gain, the vibration can be efficiently damped for both configurations. However, when the feedback gain is too high, the vibration significantly increases at the resonance frequency of the actuator, due to the the phase response of the actuator in that frequency region. Comparing Fig. 5a and Fig. 5b it can be seen that in the second case the resonance of the actuator is shifted down in frequency; so, the gain margin of the control system increases and the control performance are thus improved (the vibration of the primary structure is more damped).

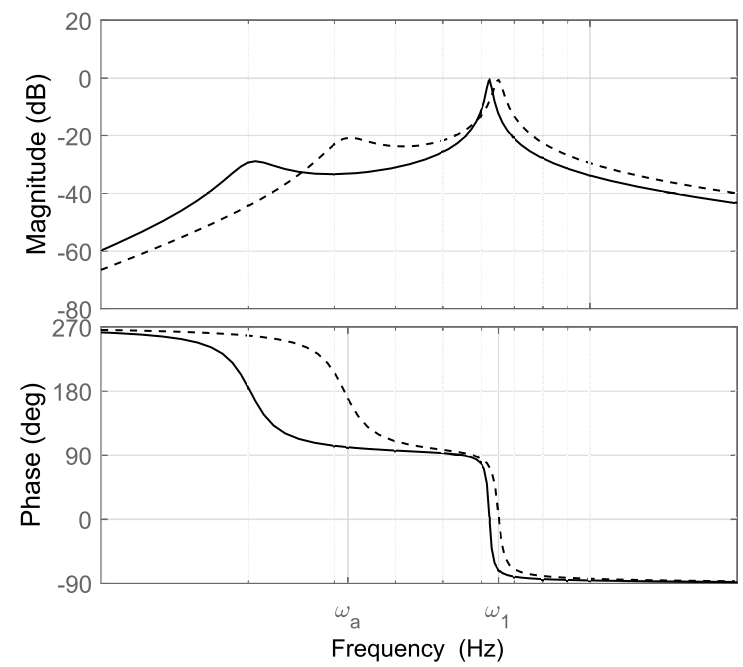

(a)

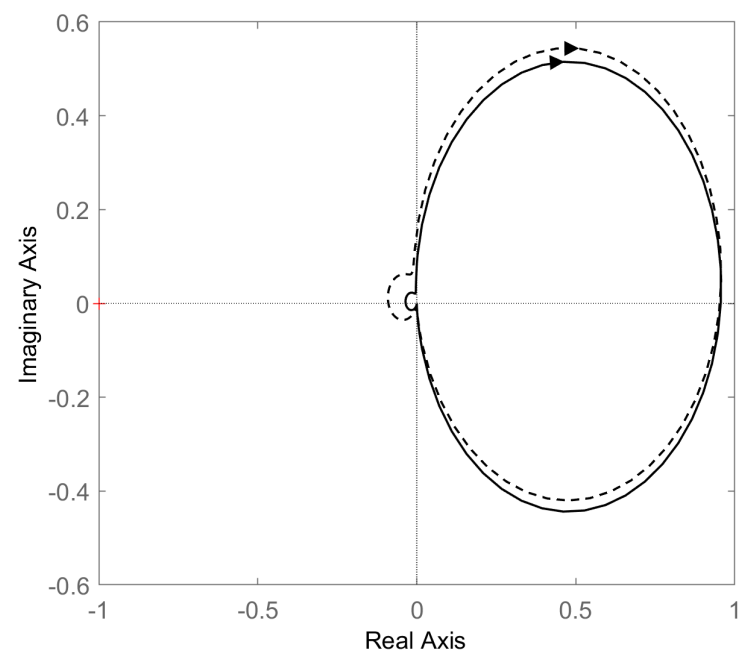

(b)

Figure 4: Bode (a) and Nyquist (b) diagrams of the open-loop Frequency Response Function without (dashed line) and with (solid line) the inerter 


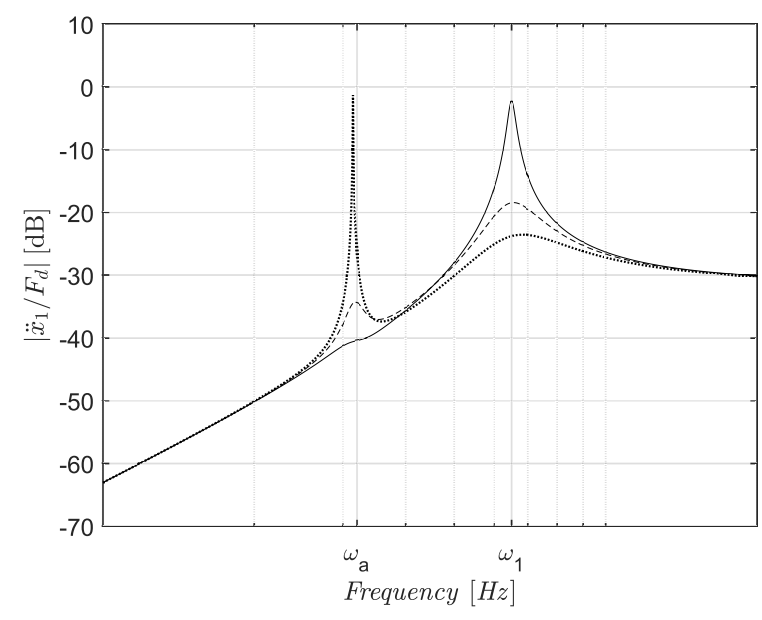

(a)

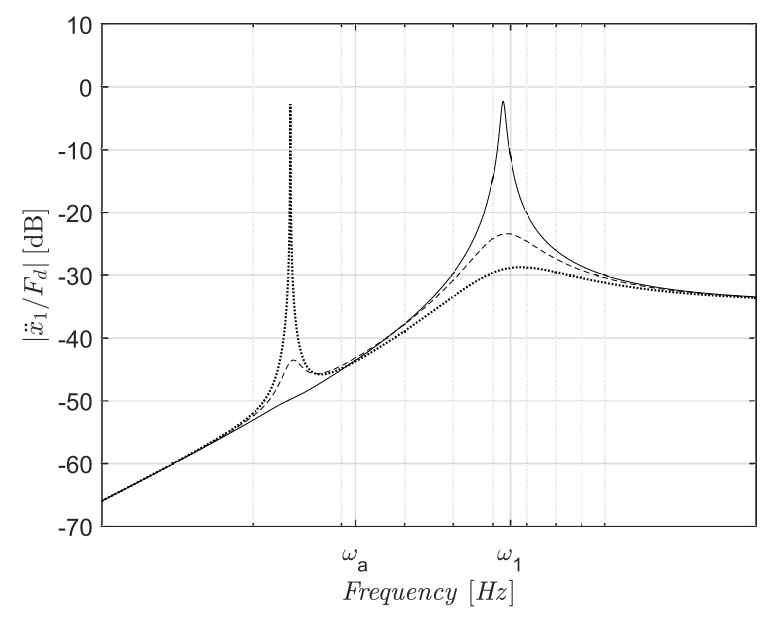

(b)

Figure 5: FRFs for zero gain (solid line) and for increasing gains (dashed and dotted lines) without (a) and with (b) the inerter

\subsection{Finite Element beam model}

This kind of procedure is then implemented into the numerical beam model. The motion equation in matrix form of the free nodal coordinates $\underline{x}_{F}$ which results from the Finite Element Analysis is written as:

$$
\left[M_{F F}\right] \underline{\ddot{x}}_{F}+\left[C_{F F}\right] \underline{\dot{x}}_{F}+\left[K_{F F}\right] \underline{x}_{F}=\underline{F}_{F}
$$

where $\underline{F}_{F}$ is the vector of equivalent nodal forces which account for the assigned time-dependent concentrated/distributed loads, related the free nodal coordinates to which they are applied. Then, in order to include the Sky-hook active force into the motion equation, it is necessary to write the control force $F_{c t r l}$ in a matrix form, as a function of the vector $\underline{\dot{x}}_{F}$ :

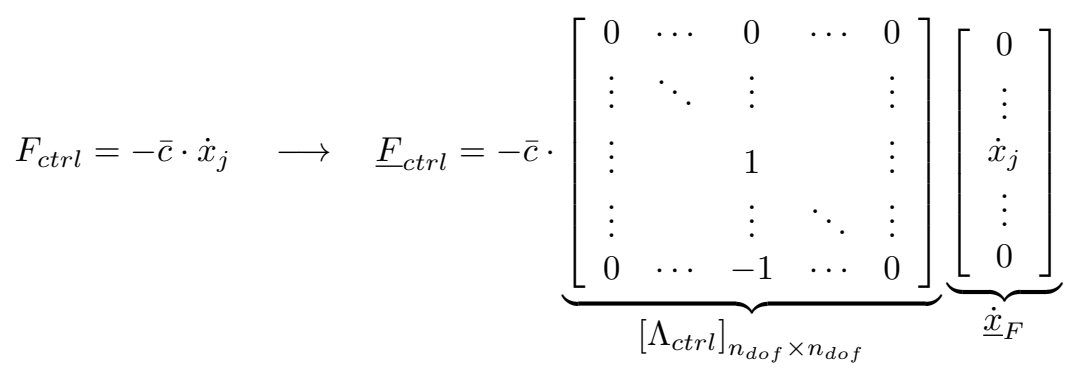

where $\dot{x}_{j}$ is the velocity of the structure at the actuator position. In the same way, also Eq. 1 can be rewritten in a matrix form, by substituting $\ddot{x}_{1}$ with the acceleration of the structure at the actuator position $\ddot{x}_{j}$ and $\ddot{x}_{a}$ with the acceleration of the suspended mass $\ddot{x}_{i}$. So, the force vector $\underline{F}_{\text {ine }}$ generated to simulate the inerter behaviour is defined as follows:

$$
F_{\text {ine }}=-b_{a}\left(\ddot{x}_{i}-\ddot{x}_{j}\right) \longrightarrow \underline{F}_{\text {ine }}=-b_{a} \cdot \underbrace{\left[\begin{array}{ccccc}
0 & \cdots & 0 & \cdots & 0 \\
\vdots & \ddots & \vdots & & \vdots \\
\vdots & & 1 & & -1 \\
\vdots & & \vdots & \ddots & \vdots \\
0 & \cdots & -1 & \cdots & 1
\end{array}\right]}_{\left[\Lambda_{\text {ine }}\right]_{n_{\text {dof }} \times n_{\text {dof }}}} \underbrace{\left[\begin{array}{c}
0 \\
\vdots \\
\dot{x}_{j} \\
\vdots \\
\dot{x}_{i}
\end{array}\right]}_{\underline{\ddot{x}}_{F}}
$$




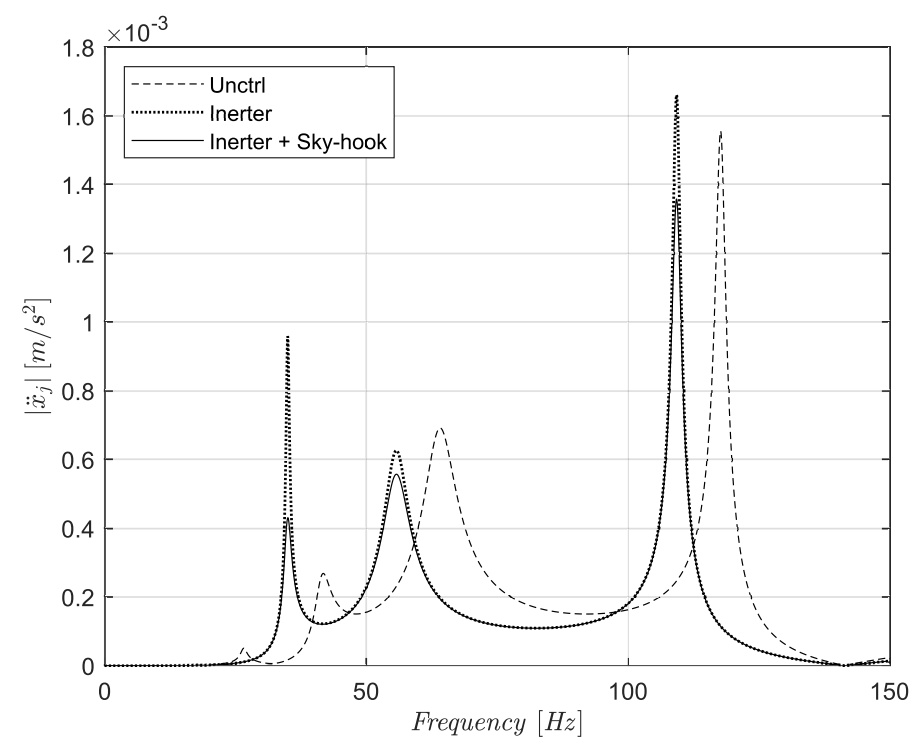

Figure 6: FRF of the uncontrolled system (dashed line) and of the system with $b_{a}>0$ and $\bar{c}=0$ (dotted line) and $\bar{c}>0$ (solid line)

At this point, the force vector $\underline{F}_{F}$ is defined as:

$$
\underline{F}_{F}=\underline{F}_{e x t}+\underline{F}_{c t r l}+\underline{F}_{i n e}
$$

where $\underline{F}_{e x t}$ is the vector of external forces acting on the system. Then, the resulting motion equation is:

$$
\left[M_{F F}\right] \underline{\ddot{x}}_{F}+\left[C_{F F}\right] \underline{\dot{x}}_{F}+\left[K_{F F}\right] \underline{x}_{F}=\underline{F}_{e x t}-\bar{c}\left[\Lambda_{c t r l}\right] \underline{\dot{x}}_{F}-b_{a}\left[\Lambda_{i n e}\right] \underline{\ddot{x}}_{F}
$$

Note that the gain $\bar{c}$ has the same meaning of the gain $\bar{c}$ used in the two degrees of freedom system.

The $\underline{F}_{c t r l}$ and $\underline{F}_{i n e}$ vectors are a linear combination of the free nodes acceleration and velocities vectors respectively; then, tehy can be brought to the left side of the equation and added to the damping and mass matrices:

$$
\left(\left[M_{F F}\right]+b_{a}\left[\Lambda_{\text {ine }}\right]\right) \ddot{\ddot{x}}_{F}+\left(\left[C_{F F}\right]+\bar{c}\left[\Lambda_{c t r l}\right]\right) \underline{\dot{x}}_{F}+\left[K_{F F}\right] \underline{x}_{F}=\underline{F}_{e x t}
$$

Now, the control law drives the actuator in order to increase the damping of the system and also to increase the mass of the actuator itself; this operation generates a sort of apparent mass which cause the down shifting of the resonance frequencies. In Fig. 6 it is possible to see the differences between three cases: a) uncontrolled system $\left(b_{a}=\bar{c}=0\right)$ b) system with $b_{a}>0$ and $\bar{c}=0$ and c) controlled system $\left(b_{a}>0, \bar{c}>0\right)$. The gain $\bar{c}$ is always kept at the same value for all the control logic tested, because in this way it possible to better compare the performances of the different cases. The unstable effect of the control has been completely rejected, but other kinds of phenomenon have appeared; the shift of the modes frequencies towards lower values generates different dynamics into the structure, and the reduction of the vibration due to the control action is very poor, especially on the fourth mode. Some considerations about this solution will be made in the next section, where the experimental results obtained with this approach are presented.

\section{EXPERIMENTAL RESULTS}

In order to implement this solution, an additional sensor is needed. Then, a new accelerometer has been mounted on the moving mass of the actuator and thus connected with the electronic board in order to provide the data needed by the control law. For the reasons explained before, this solution could be feasible and effective for vibration control of structures in which instability conditions due to the modes positions may occur. However, there is a problem which has not be taken into account in the previous discussion: in the two degrees of freedom 


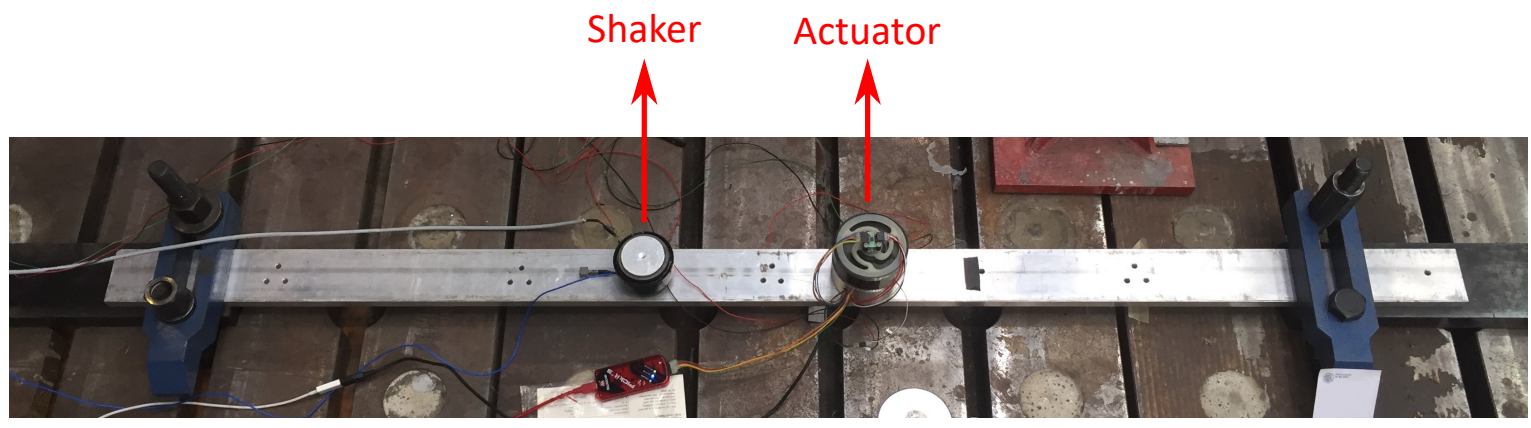

Figure 7: Experimental test rig

example no considerations are made about the replacement of the physical inerter with the "virtual" one. From a numerical point of view, there is no difference between a physical inerter and a virtual one, because its effect of the mass matrix $M$ is the same for both the cases. Nevertheless, in the real case in order to simulate its presence in the system, the control action must be set as defined by Eq. 18; this means that the command signal sent to the actuator will be given by the sum of the sky-hook damping contribution and the one related to the virtual inerter; a similar condition involves some problems related to the stroke/force saturation of the inertial actuator.

The presence of the inerter, physical or virtual, generates an apparent mass which is added to the physical mass of the actuator, in order to decrease its resonance frequency. However, by increasing the mass of the actuator, the power needed to move the suspended mass increases, and for this reason the vibration reduction with the same digital gain is lower than the other cases; this fact is confirmed by the numerical results shown in Fig. 6, where it can be seen that the controlled system curve (solid line) corresponds to a poor reduction of the vibration, especially regarding the fourth mode. So, in order to obtain a better vibration reduction with this solution, the feedback gain must be significantly increased. This fact leads to a faster transition towards the instability region, and the results in terms of damping increase are not satisfactory. In Fig. 9a are represented the FRFs of the system considering the uncontrolled case (blue line) and the controlled one (red line). In this case controlled system means that the command signal is given by the virtual inerter contribution and the Sky-hook damping contribution. This result is similar to the one depicted in Fig. 6, with a smaller shifting of the resonance frequencies (but that can be still appreciated) due to the physical limits of the actuator itself. In Fig. 9b, instead, is represented the time response of the structure acceleration with a driving frequency of the external forcing action still equal to the fourth mode one; the vibration reduction obtained with this solution is about $47 \%$ with respect to the uncontrolled case (still considering the RMS value).

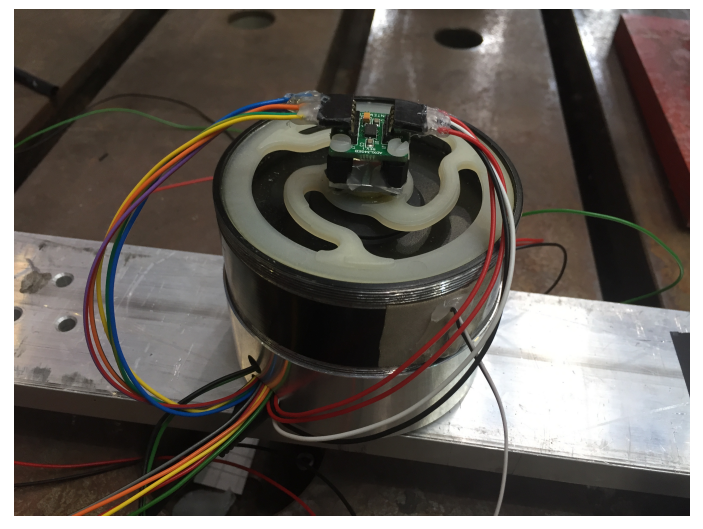

Figure 8: Detail of the accelerometer mounted on the suspended mass of the actuator 


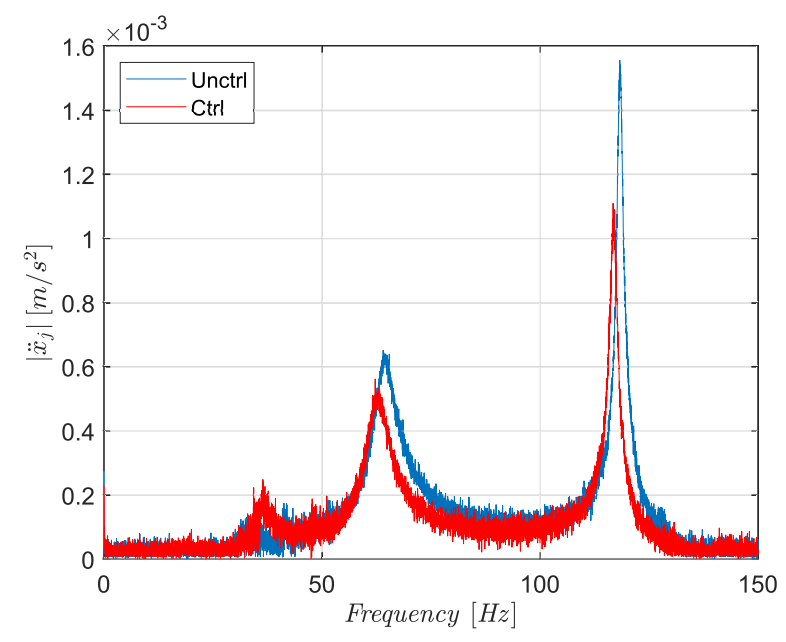

(a)
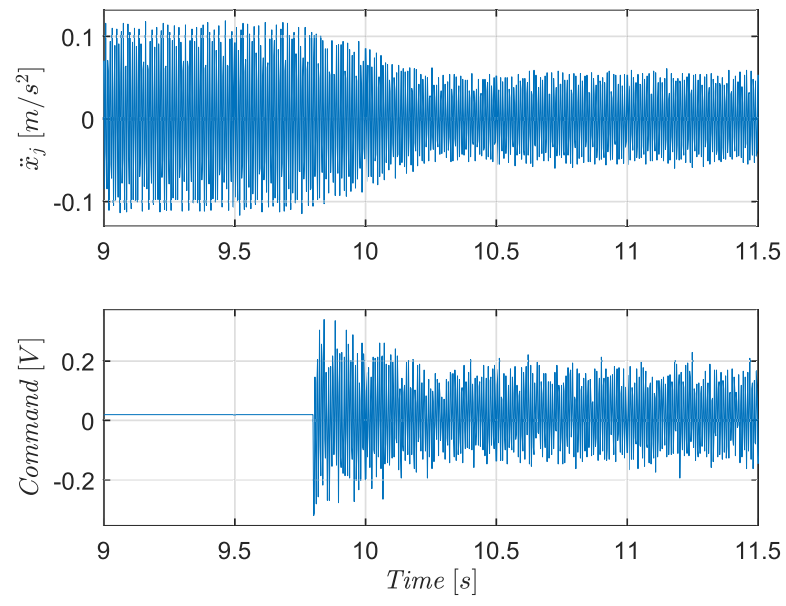

(b)

Figure 9: FFT (a) of uncontrolled (blue line) and controlled system (red line) and time response (b) of the structure excited to the fourth mode frequency

\section{CONCLUSIONS}

This work aims to investigate a possible solution which can limit instability problems related to the dynamics of inertial actuators. An inertial actuator behaves like an ideal force generator only at frequencies above its resonance frequency, while below it the force transmitted to the structure is very small and in counter-phase with respect to the control force. So, the idea is to decrease the fundamental frequency of the actuator as much as possible, in order to extend the region in which it correctly works.

This approach is carried out by using an inerter, placed in parallel with the elastic suspension of the actuator and the transducer. The physical element is replaced by the virtual one, by simulating its behaviour through the acceleration feedback which drives the actuator. The implementation of this approach into the numerical model of the beam shows that there is a strong interaction of the control unit with the other modes of the structure. The experimental results are consistent with the model, but a limited shift in frequency of the vibration modes is achieved. In this case, the presence of a vibration mode of the structure with a lower frequency with respect to the resonance frequency of the actuator makes this solution unfeasible. An important shift in frequency is required and the control unit is not able to provide it. Due to technical constraints of the actuator, the effectiveness of the control action is quite low.

A similar approach would be useful for applications in which the natural frequency of the actuator is lower than all eigenfrequencies of the structure under control, but close to the first one. In that case, the use of an inerter would lower the resonance frequency of the actuator in order to increase the gain margin, improving the stability of the control system.

\section{REFERENCES}

[1] Balas, M. J., "Feedback control of flexible systems," IEEE Transactions on Automatic Control (1978).

[2] Elliott, S. J., Serrand, M., and Gardonio, P., "Feedback stability limits for active isolation systems with reactive and inertial actuators," Journal of Vibration and Acoustics (2001).

[3] Benassi, L. and Elliott, S. J., "Active vibration isolation using an inertial actuator with local displacement feedback control," Journal of Sound and Vibration (2004).

[4] Chen, M. Z. Q., Hu, Y., Huang, L., and Chen, G., "Influence of inerter on natural frequencies of vibration systems," Journal of Sound and Vibration (2013). 
[5] Brzeski, P., Pavlovskaia, E., Kapitaniak, T., and Perlikowski, P., "The application of inerter in tuned mass absorber," International Journal of Non-Linear Mechanics (2014).

[6] Zilletti, M., "Feedback control unit with an inerter proof-mass electrodynamic actuator," Journal of Sound and Vibration (2016).

[7] Cinquemani, S. and Braghin, F., "Decentralized active vibration control in cruise ship funnels," Ocean Engineering (2017).

[8] Cinquemani, S., Cazzulani, G., Costa, A., and Resta, F., "Design of a stand-alone active damper for distributed control of vibration," (2016).

[9] Cinquemani, S. and Resta, F., "A mechanical approach to the design of IMSC for vibration suppression," Journal of Vibration and Acoustics (2013).

[10] Cazzulani, G., Resta, F., and Ripamonti, F., "Active modal tuned mass damper for smart structures," Engineering Letters (2011).

[11] Benassi, L., Elliott, S. J., and Gardonio, P., "Active vibration isolation using an inertial actuator with local force feedback control," Journal of Sound and Vibration (2004).

[12] Zimmerman, D. C. and Inman, D. J., "On the nature of the interaction between structures and proof-mass actuators," Journal of Guidance (1989).

[13] Winberg, M., Johansson, S., and Claesson, I., "Inertial mass actuators, understanding and tuning," (2004).

[14] Paulitsch, C., Gardonio, P., Elliott, S. J., Sas, P., and Boonen, R., "Design of a lightweight, electrodynamic, inertial actuator with integrated velocity sensor for active vibration control of a thin lightly-damped panel," Proceedings of the 2004 International Conference on Noise and Vibration Engineering (2004).

[15] Cinquemani, S., Resta, F., and Monguzzi, M., "Limits on the use of inertial actuators in active vibration control," (2007).

[16] Paulitsch, C., Gardonio, P., and Elliott, S. J., "Active vibration damping using an inertial, electrodynamic actuator," Journal of Vibration and Acoustics (2007).

[17] Elliott, S. J., Gardonio, P., Rafaely, B., Harris, R., and Heron, K., "Performance evaluation of a feedback active isolation system with inertial actuators," ISVR Technical Memorandum (1998).

[18] Benassi, L. and Elliott, S. J., "Global control of a vibrating plate using a feedback-controlled inertial actuator," Journal of Sound and Vibration (2005).

[19] Smith, M. C., "Synthesis of mechanical networks: the inerter," IEEE Transactions on Automatic Control (2002). 